\title{
Some Definition of Hartley-Hilbert and Fourier-Hilbert Transforms in a Quotient Space of Boehmians
}

\author{
S. K. Q. Al-Omari \\ Department of Applied Sciences, Faculty of Engineering Technology, Al-Balqa' Applied University, Amman 11134, Jordan \\ Correspondence should be addressed to S. K. Q. Al-Omari; s.k.q.alomari@fet.edu.jo
}

Received 20 May 2014; Accepted 16 August 2014; Published 6 November 2014

Academic Editor: Tepper L Gill

Copyright (C) 2014 S. K. Q. Al-Omari. This is an open access article distributed under the Creative Commons Attribution License, which permits unrestricted use, distribution, and reproduction in any medium, provided the original work is properly cited.

We investigate the Hartley-Hilbert and Fourier-Hilbert transforms on a quotient space of Boehmians. The investigated transforms are well-defined and linear mappings in the space of Boehmians. Further properties are also obtained.

\section{Introduction}

The Hilbert transform of a function $f(y)$ via the Hartley transform is defined in $[1,2]$ as

$$
\begin{aligned}
& (f)_{\mathfrak{h} \mathfrak{h}}(y) \\
& \quad=\mathfrak{h} \mathfrak{h}(f)(y) \\
& \quad=\frac{1}{\pi} \int_{0}^{\infty}\left((f)_{\mathfrak{h}}^{\mathfrak{o}}(x) \cos (x y)+(f)_{\mathfrak{h}}^{\mathfrak{e}}(x) \sin (x y)\right) d x,
\end{aligned}
$$

where $(f)_{\mathfrak{h}}^{\mathfrak{e}}$ and $(f)_{\mathfrak{h}}^{\mathfrak{D}}$ are, respectively, the even and odd components of the Hartley transform $(f)_{\mathfrak{h}}$ given as [3]

$$
(f)_{\mathfrak{h}}(x)=\int_{-\infty}^{\infty} f(y) \operatorname{cas}(x y) d y:=(f)_{\mathfrak{h}}^{\mathfrak{e}}(x)+(f)_{\mathfrak{h}}^{\mathfrak{o}}(x),
$$

where $\operatorname{cas}(x y)=\cos (x y)+\sin (x y)$

Let $f(y)$ be a casual function; that is, $f(y)=0$, for $y>0$, and then $(f)_{\mathfrak{h}}^{\mathfrak{o}}(x)$ and $(f)_{\mathfrak{h}}^{\mathfrak{e}}(x)$ are related by a Hilbert transform pair as [3]

$$
\begin{aligned}
& (f)_{\mathfrak{h}}^{\mathfrak{o}}(x)=\frac{1}{\pi} \int_{-\infty}^{\infty} \frac{(f)_{\mathfrak{h}}^{\mathfrak{e}}(y)}{x-y} d y, \\
& (f)_{\mathfrak{h}}^{\mathfrak{e}}(x)=\frac{1}{\pi} \int_{-\infty}^{\infty} \frac{(f)_{\mathfrak{h}}^{\mathfrak{o}}(y)}{x-y} d y .
\end{aligned}
$$

The Hilbert transform of $f(y)$ via the Fourier transform is defined by

$$
\begin{aligned}
& (f)_{\mathfrak{F h}}(y) \\
& \quad=\mathfrak{F} \mathfrak{h}(f)(y) \\
& \quad=\frac{1}{\pi} \int_{0}^{\infty}\left((f)_{\mathfrak{f}}^{\mathfrak{i}}(x) \cos (x y)-(f)_{\mathfrak{f}}^{\mathfrak{r}}(x) \sin (x y)\right) d x,
\end{aligned}
$$

where $(f)_{\mathfrak{f}}^{\mathfrak{r}}(x)$ and $(f)_{\mathfrak{f}}^{\mathfrak{i}}(x)$ are, respectively, the real and imaginary components of the Fourier transform given as

$$
(f)_{\mathfrak{f}}(x)=\int_{-\infty}^{\infty} f(y) e^{-i x y} d y:=(f)_{\mathfrak{f}}^{\mathfrak{r}}(x)-i(f)_{\mathfrak{i}}^{\mathfrak{i}}(x) .
$$

The Hartley transform is extended to Boehmians in [4] and to strong Boehmians in [5]. The Hartley-Hilbert and Fourier-Hilbert transforms were discussed in various spaces of distributions and spaces of Boehmians in $[1,6]$.

In this paper, $i$ aim at investigating the Hartley-Hilbert transform on the context of Boehmians. Investigating the later transform is analogous.

\section{Spaces of Quotients (Spaces of Boehmians)}

One of the most youngest generalizations of functions and more particularly of distributions is the theory of Boehmians. The idea of the construction of Boehmians was initiated by the concept of regular operators [7]. Regular operators form 
a subalgebra of the field of Mikusinski operators and they include only such functions whose support is bounded from the left. In a concrete case, the space of Boehmians contains all regular operators, all distributions, and some objects which are neither operators nor distributions.

The construction of Boehmians is similar to the construction of the field of quotients and, in some cases, it gives just the field of quotients. On the other hand, the construction is possible where there are zero divisors, such as space $\mathscr{C}$ (the space of continuous functions) with the operations of pointwise additions and convolution.

A number of integral transforms have been extended to Boehmian spaces in the recent past by many authors such as Roopkumar in [8, 9]; Mikusinski and Zayed in [10]; AlOmari and Kilicman in $[1,6,11]$; Karunakaran and Vembu [12]; Karunakaran and Roopkumar [13]; Al-Omari et al. in [14]; and many others.

For abstract construction of Boehmian spaces we refer to [14-16].

By $\circledast$ denote the Mellin-type convolution product of first kind defined by [8]

$$
(\phi \circledast \psi)(t)=\int_{0}^{\infty} z^{-1} \phi\left(t z^{-1}\right) g(z) d z .
$$

Properties of $\circledast$ are presented as follows [8]:

(i) $(\phi \circledast \psi)(t)=(\psi \circledast \phi)(t)$;

(ii) $((\phi+\psi) \circledast \varphi)(t)=(\phi \circledast \varphi)(t)+(\psi \circledast \varphi)(t)$;

(iii) $(\alpha \phi \circledast \psi)(t)=\alpha(\psi \circledast \phi)(t) ; \alpha$ is complex number;

(iv) $((\phi \circledast \psi) \circledast \varphi)(t)=(\phi \circledast(\psi \circledast \varphi))(t)$.

By $*$, we denote the convolution product defined by

$$
(\phi * \psi)(y)=\int_{0}^{\infty} z^{-1} \phi\left(y z^{-1}\right) \psi(z) d z .
$$

By $\boldsymbol{\vartheta}\left(\mathbb{R}_{+}\right)$denote the space of test functions of bounded supports defined on $\mathbb{R}_{+}$that vanish more rapidly than every power of $x$ as $x \rightarrow \infty$. Denote by $\boldsymbol{\vartheta}_{1}$ the subset of $\boldsymbol{\vartheta}\left(\mathbb{R}_{+}\right)$ defined by

$$
\boldsymbol{\vartheta}_{1}=\left\{\varphi \in \boldsymbol{\vartheta}\left(\mathbb{R}_{+}\right): \int_{0}^{\infty} \varphi(x) d x=1\right\} .
$$

Let $\Delta$ be the set of delta sequences satisfying the following properties:

(1): $\left\{\delta_{n}\right\} \in \boldsymbol{\vartheta}\left(\mathbb{R}_{+}\right)$;

(2): $\int_{0}^{\infty} \delta_{n}(x) d x=1, n \in \mathbb{N}$;

(3): $\int_{0}^{\infty}\left|\delta_{n}(x)\right| d x<\infty, n \in \mathbb{N}$;

(4): $\operatorname{supp} \delta_{n}(x) \subseteq\left[a, b_{n}\right], 0<a_{n}<b_{n}$ and $a_{n}, b_{n} \rightarrow 0$ as $n \rightarrow \infty$, where

$$
\operatorname{supp} \delta_{n}(x)=\left\{x \in \mathbb{R}_{+}: \delta_{n}(x) \neq 0, \forall n \in \mathbb{N}\right\} .
$$

Now we establish the following theorem.
Theorem 1. Let $\phi, \psi \in \mathfrak{\vartheta}\left(\mathbb{R}_{+}\right)$; then one has $(\phi \circledast \psi)_{\mathfrak{h G}}(y)=$ $\left(\phi_{\mathfrak{h y}} * \psi\right)(y)$.

Proof. Let $\phi$ and $\psi$ be in $\boldsymbol{\vartheta}\left(\mathbb{R}_{+}\right)$; then we have

$$
\begin{aligned}
&(\phi \circledast \psi)_{\mathfrak{h \mathfrak { h }}}(y)=\int_{0}^{\infty}((\phi \circledast \psi)_{\mathfrak{h}}^{\mathfrak{o}}(x) \cos (x y) \\
&\left.+(\phi \circledast \psi)_{\mathfrak{h}}^{\mathfrak{e}}(x) \sin (x y)\right) d x \\
& \text { i.e. }=\int_{0}^{\infty}\left(\int_{0}^{\infty}(\phi \circledast \psi)(t) \sin (x t) d t \cos (x y)\right. \\
& \quad+\int_{0}^{\infty}(\phi \circledast \psi)(t) \cos (x t) d t \\
&\quad \times \sin (x y)) d x, \\
& \text { i.e. }=\int_{0}^{\infty}\left(\int _ { 0 } ^ { \infty } \left(\int _ { 0 } ^ { \infty } \left(\phi\left(t z^{-1}\right) \sin (x t) \cos (x y)\right.\right.\right. \\
&+\phi\left(t z^{-1}\right) \cos (x t) \\
&\times \sin (x y)) d t) d x) \\
& \times \psi(z) z^{-1} d z .
\end{aligned}
$$

By the change of variables $t z^{-1}=w$, we get that

$$
\begin{array}{r}
(\phi \circledast \psi)_{\mathfrak{h h}}(y)=\int_{0}^{\infty}\left(\int _ { 0 } ^ { \infty } \left(\int_{0}^{\infty}(\phi(w) \sin (x z w) \cos (x y)\right.\right. \\
+\phi(w) \cos (x z w) \\
\times \sin (x y)) d w) d x) \\
\times \psi(z) z d z, \\
\text { i.e. }=\int_{0}^{\infty}\left(\int _ { 0 } ^ { \infty } \left(\phi_{\mathfrak{h}}^{\mathfrak{j}}(x z) \cos (x y)\right.\right. \\
\times \psi(z) z d z .
\end{array}
$$

Once again, by the change of variables $x z=r$, we get

$$
(\phi \circledast \psi)_{\mathfrak{h y ~}}(y)=\int_{0}^{\infty} z^{-1} \phi_{\mathfrak{h h}}\left(y z^{-1}\right) \psi(z) d z .
$$

This completes the proof of the theorem.

Now, we establish the following theorem.

Theorem 2. Let $\phi$, $\psi$, and $\theta$ be test functions in $\boldsymbol{\vartheta}\left(\mathbb{R}_{+}\right)$; then $\phi *(\psi \circledast \theta)=(\phi * \psi) * \theta$. 
Proof. Let $y>0$ be given. Then, from (6) and (7), we have

$$
\begin{gathered}
(\phi *(\psi \circledast \theta))(y)=\int_{0}^{\infty} z^{-1} \phi\left(y z^{-1}\right)(\psi \circledast \theta)(z) d z \\
\text { i.e. }=\int_{0}^{\infty} z^{-1} \phi\left(y z^{-1}\right)\left(\int_{0}^{\infty} t^{-1} \psi\left(z t^{-1}\right) \theta(t) d t\right) d z \\
\text { i.e. }=\int_{0}^{\infty} z^{-1} \phi\left(y z^{-1}\right)(\psi * \theta)(z) d z
\end{gathered}
$$

Hence, the theorem is completely proved.

Theorem 3. Let $\varphi, \psi \in \boldsymbol{\vartheta}_{1}$; then $\varphi \circledast \psi=\psi \circledast \varphi$.

Proof. Proof of this theorem follows from technique similar to that of Theorem 2. Therefore, we omit the details.

Theorem 4. Let $\phi_{1}, \phi_{2} \in \boldsymbol{\vartheta}\left(\mathbb{R}_{+}\right)$and $\varphi_{1}, \varphi_{2} \in \boldsymbol{\vartheta}_{1}$; then the following hold:

(1) $\left(\phi_{1}+\phi_{2}\right) * \varphi_{1}=\phi_{1} * \varphi_{1}+\phi_{2} * \varphi_{1}$;

(2) if $\phi_{n} \rightarrow \phi \in \mathfrak{\vartheta}\left(\mathbb{R}_{+}\right)$as $n \rightarrow \infty$, then $\phi_{n} * \varphi \rightarrow \phi * \varphi$ as $n \rightarrow \infty$

(3) $\phi_{1} *\left(\varphi_{1} \circledast \varphi_{2}\right)=\left(\phi_{1} * \varphi_{1}\right) * \varphi_{2}$;

(4) $\phi_{1} * \varphi_{1} \in \boldsymbol{\vartheta}\left(\mathbb{R}_{+}\right)$.

Proof. Proof of (1) and (2) follows from elementary integral calculus. Proof of (3) is already obtained in Theorem 2. Proof of (4) is obvious by the properties of the product (7). Further details are, thus, avoided.

This completes the proof of the theorem.

Theorem 5. Let $\phi \in \mathfrak{\vartheta}\left(\mathbb{R}_{+}\right)$and $\left\{\delta_{n}\right\} \in \Delta$; then $\phi * \delta_{n} \rightarrow \phi$ as $n \rightarrow \infty$.

Proof. Let $K$ be a compact set containing the support of $\phi$; then, by Property 2 of delta sequences, we have

$$
\begin{aligned}
& \left|\mathscr{D}_{y}^{k}\left(\phi * \delta_{n}-\phi\right)(y)\right| \\
& \quad \leq \int_{K}\left|\mathscr{D}_{y}^{k}\left(z^{-1} \phi\left(y z^{-1}\right)-\phi(y)\right)\right|\left|\delta_{n}(y)\right| d z .
\end{aligned}
$$

Hence, (14) goes to zero as $n \rightarrow \infty$.

Hence the theorem is completely proved.

The Boehmian space $\mathscr{B}\left(\boldsymbol{\vartheta},\left(\boldsymbol{\vartheta}_{1}, \circledast\right), *, \Delta\right)$ is constructed.

The sum of two Boehmians and multiplication by a scalar can be defined in a natural way:

$$
\begin{gathered}
{\left[\frac{\left\{f_{n}\right\}}{\left\{\omega_{n}\right\}}\right]+\left[\frac{\left\{g_{n}\right\}}{\left\{\psi_{n}\right\}}\right]=\left[\frac{\left\{f_{n} * \psi_{n}+g_{n} * \omega_{n}\right\}}{\left\{\omega_{n} \circledast \psi_{n}\right\}}\right],} \\
\alpha\left[\frac{\left\{f_{n}\right\}}{\left\{\omega_{n}\right\}}\right]=\left[\alpha \frac{\left\{f_{n}\right\}}{\left\{\omega_{n}\right\}}\right]=\left[\frac{\left\{\alpha f_{n}\right\}}{\left\{\omega_{n}\right\}}\right],
\end{gathered}
$$

where $\alpha \in \mathbb{C}$, the field of complex numbers.
The operation $*$ and the differentiation are defined by

$$
\begin{gathered}
{\left[\frac{\left\{f_{n}\right\}}{\left\{\omega_{n}\right\}}\right] *\left[\frac{\left\{g_{n}\right\}}{\left\{\psi_{n}\right\}}\right]=\left[\frac{\left\{f_{n} * g_{n}\right\}}{\left\{\omega_{n} \circledast \psi_{n}\right\}}\right],} \\
\mathscr{D}^{\alpha}\left[\frac{\left\{f_{n}\right\}}{\left\{\omega_{n}\right\}}\right]=\left[\frac{\left\{\mathscr{D}^{\alpha} f_{n}\right\}}{\left\{\omega_{n}\right\}}\right] .
\end{gathered}
$$

Similarly, the space $\mathscr{B}\left(\boldsymbol{\vartheta},\left(\boldsymbol{\vartheta}_{1}, \circledast\right), \circledast, \Delta\right)$ can be proved.

The sum of two Boehmians and multiplication by a scalar can be defined in a natural way:

$$
\begin{gathered}
{\left[\frac{\left\{f_{n}\right\}}{\left\{\omega_{n}\right\}}\right]+\left[\frac{\left\{g_{n}\right\}}{\left\{\psi_{n}\right\}}\right]=\left[\frac{\left\{f_{n} \circledast \psi_{n}+g_{n} \circledast \omega_{n}\right\}}{\left\{\omega_{n} \circledast \psi_{n}\right\}}\right],} \\
\alpha\left[\frac{\left\{f_{n}\right\}}{\left\{\omega_{n}\right\}}\right]=\left[\alpha \frac{\left\{f_{n}\right\}}{\left\{\omega_{n}\right\}}\right]=\left[\frac{\left\{\alpha f_{n}\right\}}{\left\{\omega_{n}\right\}}\right] .
\end{gathered}
$$

The operation $*$ and the differentiation are defined by

$$
\begin{gathered}
{\left[\frac{\left\{f_{n}\right\}}{\left\{\omega_{n}\right\}}\right] \circledast\left[\frac{\left\{g_{n}\right\}}{\left\{\psi_{n}\right\}}\right]=\left[\frac{\left\{f_{n} \circledast g_{n}\right\}}{\left\{\omega_{n} \circledast \psi_{n}\right\}}\right],} \\
\mathscr{D}^{\alpha}\left[\frac{\left\{f_{n}\right\}}{\left\{\omega_{n}\right\}}\right]=\left[\frac{\left\{\mathscr{D}^{\alpha} f_{n}\right\}}{\left\{\omega_{n}\right\}}\right] .
\end{gathered}
$$

As next, let $\phi \in \boldsymbol{\vartheta}\left(\mathbb{R}_{+}\right)$; then $(\phi)_{\mathfrak{h h}}(y) \in \boldsymbol{\vartheta}\left(\mathbb{R}_{+}\right)$.

For some details, we by definitions have that

$$
\begin{aligned}
& \left|\mathscr{D}_{y}^{k}(\phi)_{\mathfrak{h} \mathfrak{h}}(y)\right| \\
& \leq \frac{1}{\pi} \int_{0}^{\infty} \mathscr{D}_{y}^{k}\left(\left|\left((\phi)_{\mathfrak{h}}^{\mathfrak{o}}(x) \cos (x y)+(\phi)_{\mathfrak{h}}^{\mathfrak{e}}(x) \sin (x y)\right)\right|\right) d x \\
& \leq \frac{1}{\pi} \int_{0}^{\infty}\left|x^{k}\right|\left|(\phi)_{\mathfrak{h}}^{\mathfrak{o}}(x)\right| d x+\frac{1}{\pi} \int_{0}^{\infty}\left|x^{k}\right|\left|(\phi)_{\mathfrak{h}}^{\mathfrak{e}}(x)\right| d x .
\end{aligned}
$$

But since

$$
\frac{1}{\pi} \int_{0}^{\infty}\left|x^{k}\right|\left|(\phi)_{\mathfrak{h}}^{\mathfrak{o}}(x)\right| d x=\frac{1}{\pi} \int_{0}^{\infty} \int_{a}^{b}\left|x^{k}\right||\phi(y)| d y d x
$$

$$
\longrightarrow 0
$$

for every power of $x$ as $x \rightarrow \infty$, where $[a, b]$ is a compact set containing the support of $\phi$, similarly we see that

$$
\frac{1}{\pi} \int_{0}^{\infty}\left|x^{k}\right|\left|(\phi)_{\mathfrak{h}}^{\mathfrak{e}}(x)\right| d x \longrightarrow 0,
$$

for every power of $x$ as $x \rightarrow \infty$. Therefore, we get that $(\phi)_{\mathfrak{h h}}(y) \in \boldsymbol{\vartheta}\left(\mathbb{R}_{+}\right)$.

\section{Hartley-Hilbert Transform of Quotients}

Let $\left[\left\{\phi_{n}\right\} /\left\{\delta_{n}\right\}\right] \in \mathscr{B}\left(\boldsymbol{\vartheta},\left(\boldsymbol{\vartheta}_{1}, \circledast\right), \circledast, \Delta\right)$; then we define the extended Hartley-Hilbert transform of $\left[\left\{\phi_{n}\right\} /\left\{\delta_{n}\right\}\right]$ as

$$
\left(\left[\frac{\left\{\phi_{n}\right\}}{\left\{\delta_{n}\right\}}\right]\right)_{\mathfrak{h h}}=\left[\frac{\left\{\left(\phi_{n}\right)_{\mathfrak{h h}}\right\}}{\left\{\delta_{n}\right\}}\right],
$$

in the quotient space $\mathscr{B}\left(\boldsymbol{\vartheta},\left(\boldsymbol{\vartheta}_{1}, \circledast\right), *, \Delta\right)$. 
Theorem 6. The operator $\left(\left[\left\{\phi_{n}\right\} /\left\{\delta_{n}\right\}\right]\right)_{\mathfrak{h h}}$ is well defined and linear from $\mathscr{B}\left(\boldsymbol{\vartheta},\left(\boldsymbol{\vartheta}_{1}, \circledast\right), \circledast, \Delta\right)$ into $\mathscr{B}\left(\boldsymbol{\vartheta},\left(\boldsymbol{\vartheta}_{1}, \circledast\right), *, \Delta\right)$.

Proof. We show that $\left(\left[\left\{\phi_{n}\right\} /\left\{\delta_{n}\right\}\right]\right)_{\mathfrak{h} \mathfrak{h}}$ is well defined.

Let $\left[\left\{\psi_{n}\right\} /\left\{\mu_{n}\right\}\right]=\left[\left\{\xi_{n}\right\} /\left\{\epsilon_{n}\right\}\right]$ in $\mathscr{B}\left(\boldsymbol{\vartheta},\left(\boldsymbol{\vartheta}_{1}, \circledast\right), \circledast, \Delta\right)$; then, by the concept of quotients of the space $\mathscr{B}\left(\boldsymbol{\vartheta},\left(\boldsymbol{\vartheta}_{1}, \circledast\right), \circledast, \Delta\right)$ we have

$$
\psi_{n} \circledast \epsilon_{m}=\xi_{m} \circledast \mu_{n}
$$

Hence, by Theorem 1, we get from (23) that

$$
\left(\psi_{n}\right)_{\mathfrak{h} \mathfrak{h}} * \epsilon_{m}=\left(\xi_{m}\right)_{\mathfrak{h} \mathfrak{h}} * \mu_{n} .
$$

Hence, from (24), it follows that

$$
\frac{\left\{\left(\psi_{n}\right)_{\mathfrak{h} \mathfrak{h}}\right\}}{\left\{\mu_{n}\right\}} \sim \frac{\left\{\left(\xi_{n}\right)_{\mathfrak{h} \mathfrak{h}}\right\}}{\left\{\epsilon_{n}\right\}},
$$

in $\mathscr{B}\left(\boldsymbol{\vartheta},\left(\boldsymbol{\vartheta}_{1}, \circledast\right), *, \Delta\right)$.

Therefore,

$$
\left[\frac{\left\{\left(\psi_{n}\right)_{\mathfrak{h h}}\right\}}{\left\{\mu_{n}\right\}}\right]=\left[\frac{\left\{\left(\xi_{n}\right)_{\mathfrak{h} \mathfrak{h}}\right\}}{\left\{\epsilon_{n}\right\}}\right],
$$

in $\mathscr{B}\left(\boldsymbol{\vartheta},\left(\boldsymbol{\vartheta}_{1}, \circledast\right), *, \Delta\right)$.

That is,

$$
\left(\left[\frac{\left\{\psi_{n}\right\}}{\left\{\mu_{n}\right\}}\right]\right)_{\mathfrak{h} \mathfrak{h}}=\left(\left[\frac{\left\{\xi_{n}\right\}}{\left\{\epsilon_{n}\right\}}\right]\right)_{\mathfrak{h} \mathfrak{h}} .
$$

To show that $\left(\left[\left\{\phi_{n}\right\} /\left\{\delta_{n}\right\}\right]\right)_{\mathfrak{h h}}$ is linear, let $\left[\left\{f_{n}\right\} /\left\{\epsilon_{n}\right\}\right]$, $\left[\left\{g_{n}\right\} /\left\{\tau_{n}\right\}\right] \in \mathscr{B}\left(\boldsymbol{\vartheta},\left(\boldsymbol{\vartheta}_{1}, \circledast\right), \circledast, \Delta\right), \kappa, \eta \in \mathbb{R}$; then

$$
\begin{aligned}
\left(\kappa\left[\frac{\left\{f_{n}\right\}}{\left\{\epsilon_{n}\right\}}\right]+\eta\left[\frac{\left\{g_{n}\right\}}{\left\{\tau_{n}\right\}}\right]\right)_{\mathfrak{h h}}=\left(\left[\frac{\kappa\left\{f_{n}\right\}}{\left\{\epsilon_{n}\right\}}\right]+\left[\frac{\eta\left\{g_{n}\right\}}{\left\{\tau_{n}\right\}}\right]\right)_{\mathfrak{h h}}, \\
\text { i.e. }=\left(\left[\frac{\left\{\left(\kappa f_{n}\right) \circledast \tau_{n}+\left(\eta g_{n}\right) \circledast \epsilon_{n}\right\}}{\left\{\epsilon_{n} \circledast \tau_{n}\right\}}\right]\right)_{\mathfrak{h h}}, \\
\text { i.e. }=\left[\frac{\left\{\left(\left(\kappa f_{n}\right) \circledast \tau_{n}+\left(\eta g_{n}\right) \circledast \epsilon_{n}\right)_{\mathfrak{h h}}\right\}}{\left\{\epsilon_{n} \circledast \tau_{n}\right\}}\right] .
\end{aligned}
$$

Linearity of the classical Hartley-Hilbert transform implies

$$
\begin{aligned}
\left(\kappa\left[\frac{\left\{f_{n}\right\}}{\left\{\epsilon_{n}\right\}}\right]+\eta\left[\frac{\left\{g_{n}\right\}}{\left\{\tau_{n}\right\}}\right]\right)_{\mathfrak{h h}} \\
\quad=\left[\frac{\kappa\left\{\left(f_{n} \circledast \tau_{n}\right)_{\mathfrak{h} \mathfrak{h}}\right\}+\eta\left\{\left(g_{n} \circledast \epsilon_{n}\right)_{\mathfrak{h} \mathfrak{h}}\right\}}{\left\{\epsilon_{n}\right\} \circledast\left\{\tau_{n}\right\}}\right] .
\end{aligned}
$$

Theorem 1 gives

$$
\begin{aligned}
\left(\kappa\left[\frac{\left\{f_{n}\right\}}{\left\{\epsilon_{n}\right\}}\right]+\eta\left[\frac{\left\{g_{n}\right\}}{\left\{\tau_{n}\right\}}\right]\right)_{\mathfrak{h h}} \\
\quad=\left[\frac{\kappa\left\{\left(f_{n}\right)_{\mathfrak{h h}}\right\} *\left\{\tau_{n}\right\}+\eta\left\{\left(g_{n}\right)_{\mathfrak{h h}}\right\} *\left\{\epsilon_{n}\right\}}{\left\{\epsilon_{n}\right\} \circledast\left\{\tau_{n}\right\}}\right] .
\end{aligned}
$$

Thus, by addition of Boehmians

$$
\begin{aligned}
& \left(\kappa\left[\frac{\left\{f_{n}\right\}}{\left\{\epsilon_{n}\right\}}\right]+\eta\left[\frac{\left\{g_{n}\right\}}{\left\{\tau_{n}\right\}}\right]\right)_{\mathfrak{h h}} \\
& =\left[\frac{\kappa\left\{\left(f_{n}\right)_{\mathfrak{h} \mathfrak{h}}\right\}}{\left\{\epsilon_{n}\right\}}\right]+\left[\frac{\eta\left\{\left(g_{n}\right)_{\mathfrak{h} \mathfrak{h}}\right\}}{\left\{\tau_{n}\right\}}\right] \text {. }
\end{aligned}
$$

Hence,

$$
\begin{aligned}
\left(\kappa\left[\frac{\left\{f_{n}\right\}}{\left\{\epsilon_{n}\right\}}\right]+\eta\left[\frac{\left\{g_{n}\right\}}{\left\{\tau_{n}\right\}}\right]\right)_{\mathfrak{h} \mathfrak{h}} \\
\quad=\kappa\left[\frac{\left\{\left(f_{n}\right)_{\mathfrak{h} \mathfrak{h}\}}\right]+\eta\left[\frac{\left\{\left(g_{n}\right)_{\mathfrak{h} \mathfrak{h}}\right\}}{\left\{\epsilon_{n}\right\}}\right] .}{\left\{\tau_{n}\right\}}\right] .
\end{aligned}
$$

The proof of the theorem is completed.

Theorem 7. The necessary and sufficient condition for $\left[\left\{g_{n}\right\} /\left\{\psi_{n}\right\}\right] \in \mathscr{B}\left(\boldsymbol{\vartheta},\left(\boldsymbol{\vartheta}_{1}, \circledast\right), *, \Delta\right)$ to be in the range of $\left(\left[\left\{g_{n}\right\} /\left\{\psi_{n}\right\}\right]\right)_{\mathfrak{h h}}$ is that $g_{n}$ belongs to range of the classical $\mathfrak{h} \mathfrak{h}$, for every $n \in \mathbb{N}$.

Proof. Let $\left[\left\{g_{n}\right\} /\left\{\psi_{n}\right\}\right]$ be in the range of $\left(\left[\left\{g_{n}\right\} /\left\{\psi_{n}\right\}\right]\right)_{\mathfrak{h} \mathfrak{h} \text {; }}$ then of course $g_{n}$ belongs to the range of the classical $\mathfrak{h} \mathfrak{h}, \forall n \in$ $\mathbb{N}$.

To establish the converse, let $g_{n}$ be in the range of $\mathfrak{h} \mathfrak{h}, \forall n \in$ $\mathbb{N}$. Then there is $\phi_{n} \in \mathfrak{\vartheta}\left(\mathbb{R}_{+}\right)$such that $\mathfrak{h} \mathfrak{h} \phi_{n}=g_{n}, n \in \mathbb{N}$.

Since $\left[\left\{g_{n}\right\} /\left\{\psi_{n}\right\}\right] \in \mathscr{B}\left(\boldsymbol{\vartheta},\left(\boldsymbol{\vartheta}_{1}, \circledast\right), *, \Delta\right)$, we get $g_{n} *$ $\psi_{m}=g_{m} * \psi_{n}, \forall m, n \in \mathbb{N}$.

Therefore, Theorem 1 yields

$$
\mathfrak{h} \mathfrak{h}\left(\phi_{n} \circledast \psi_{m}\right)=\mathfrak{h} \mathfrak{h}\left(\phi_{m} \circledast \psi_{n}\right), \quad \forall m, n \in \mathbb{N}
$$

where $\phi_{n} \in \boldsymbol{\vartheta}\left(\mathbb{R}_{+}\right)$and $\left\{\psi_{n}\right\} \in \Delta, \forall n \in \mathbb{N}$.

Thus, $\phi_{n} \circledast \psi_{m}=\phi_{m} \circledast \psi_{n}, m, n \in \mathbb{N}$.

Hence,

$$
\begin{gathered}
{\left[\frac{\left\{\phi_{n}\right\}}{\left\{\psi_{n}\right\}}\right] \in \mathscr{B}\left(\boldsymbol{\vartheta},\left(\boldsymbol{\vartheta}_{1}, \circledast\right), \circledast, \Delta\right),} \\
\left(\left[\frac{\left\{\phi_{n}\right\}}{\left\{\psi_{n}\right\}}\right]\right)_{\mathfrak{h h}}=\left[\frac{\left\{g_{n}\right\}}{\left\{\psi_{n}\right\}}\right] .
\end{gathered}
$$

The theorem is, therefore, completely proved.

Theorem 8. The transform $\left(\left[\left\{\phi_{n}\right\} /\left\{\psi_{n}\right\}\right]\right)_{\mathfrak{h G}}$ is consistent with $\mathfrak{h} \mathfrak{h}: \boldsymbol{\vartheta}\left(\mathbb{R}_{+}\right) \rightarrow \boldsymbol{\vartheta}\left(\mathbb{R}_{+}\right)$. 
Proof. For every $\phi \in \boldsymbol{\vartheta}\left(\mathbb{R}_{+}\right)$, let $\beta \in \mathscr{B}\left(\boldsymbol{\vartheta},\left(\boldsymbol{\vartheta}_{1}, \circledast\right), \circledast, \Delta\right)$ be its representative; then we have $\beta=\left[\phi \circledast\left\{\psi_{n}\right\} /\left\{\psi_{n}\right\}\right], \forall n \in \mathbb{N}$, $\left\{\psi_{n}\right\} \in \Delta$. For all $n \in \mathbb{N}$ it is clear that $\left\{\psi_{n}\right\}$ is independent from the representative.

By Theorem 1 we have

$$
\begin{aligned}
(\beta)_{h h} & =\left(\left[\frac{\phi \circledast\left\{\psi_{n}\right\}}{\left\{\psi_{n}\right\}}\right]\right)_{\mathfrak{h h}} \\
& =\left[\frac{\mathfrak{h} \mathfrak{h}\left(\phi \circledast\left\{\psi_{n}\right\}\right)}{\left\{\psi_{n}\right\}}\right]=\left[\frac{\mathfrak{h} \mathfrak{h} \phi *\left\{\psi_{n}\right\}}{\left\{\psi_{n}\right\}}\right],
\end{aligned}
$$

which is the representative of $\mathfrak{h} h \phi$ in the space $\mathfrak{\vartheta}\left(\mathbb{R}_{+}\right)$.

Hence the proof of this theorem is completed.

\section{Conflict of Interests}

The author declares that there is no conflict of interests regarding the publication of this paper.

\section{Acknowledgment}

The author expresses his gratitude to the referees for valuable suggestions.

\section{References}

[1] S. K. Q. Al-Omari and A. Kılıçman, "Some remarks on the extended Hartley-Hilbert and Fourier-Hilbert transforms of boehmians," Abstract and Applied Analysis, vol. 2013, Article ID 348701, 6 pages, 2013.

[2] N. Sundararajan and Y. Srinivas, "Fourier-Hilbert versus Hartley-Hilbert transforms with some geophysical applications," Journal of Applied Geophysics, vol. 71, no. 4, pp. 157-161, 2010.

[3] R. P. Millane, "Analytical properties of the Hartley transform and their implications," Proceedings of the IEEE, vol. 82, no. 3, pp. 413-428, 1994.

[4] S. K. Al-Omari and A. Kılıçman, "On diffraction Fresnel transforms for Boehmians," Abstract and Applied Analysis, vol. 2011, Article ID 712746, 11 pages, 2011.

[5] S. K. Al-Omari, "Hartley transforms on a certain space of generalized functions," Georgian Mathematical Journal, vol. 20, no. 3, pp. 415-426, 2013.

[6] S. K. Q. Al-Omari and A. Kilicman, "On the generalized Hartley-Hilbert and Fourier-Hilbert transforms," Advances in Difference Equations, vol. 2012, article 232, 2012.

[7] T. K. Boehme, “The support of Mikusiński operators," Transactions of the American Mathematical Society, vol. 176, pp. 319-334, 1973.

[8] R. Roopkumar, "Mellin transform for Boehmians," Bulletin of the Institute of Mathematics: Academia Sinica: New Series, vol. 4, no. 1, pp. 75-96, 2009.

[9] R. Roopkumar, "Generalized Radon transform," Rocky Mountain Journal of Mathematics, vol. 36, no. 4, pp. 1375-1390, 2006.

[10] P. Mikusinski and A. Zayed, "The Radon transform of Boehmians," Proceedings of the American Mathematical Society, vol. 118, no. 2, pp. 561-570, 1993.
[11] S. K. Al-Omari and A. Kilicman, "Note on Boehmians for class of optical Fresnel wavelet transforms," Journal of Function Spaces and Applications, vol. 2021, Article ID 405368, 14 pages, 2012.

[12] V. Karunakaran and R. Vembu, "Hilbert transform on periodic boehmians," Houston Journal of Mathematics, vol. 29, no. 2, pp. 437-454, 2003.

[13] V. Karunakaran and R. Roopkumar, "Operational calculus and Fourier transform on Boehmians," Colloquium Mathematicum, vol. 102, no. 1, pp. 21-32, 2005.

[14] S. K. Al-Omari, D. Loonker, P. K. Banerji, and S. L. Kalla, "Fourier sine (cosine) transform for ultradistributions and their extensions to tempered and ultraBoehmian spaces," Integral Transforms and Special Functions, vol. 19, no. 6, pp. 453-462, 2008.

[15] P. Mikusinski, "Tempered Boehmians and ultradistributions," Proceedings of the American Mathematical Society, vol. 123, no. 3, pp. 813-817, 1995.

[16] R. S. Pathak, Integral Transforms of Generalized Functions and Their Applications, Gordon and Breach Science, Amsterdam, The Netherlands, 1997. 


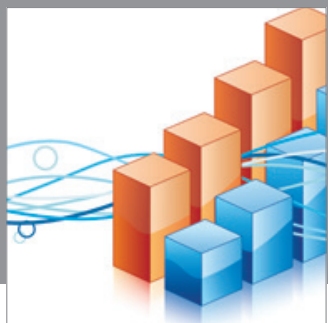

Advances in

Operations Research

mansans

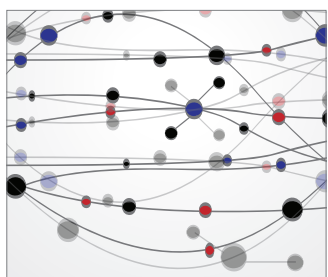

The Scientific World Journal
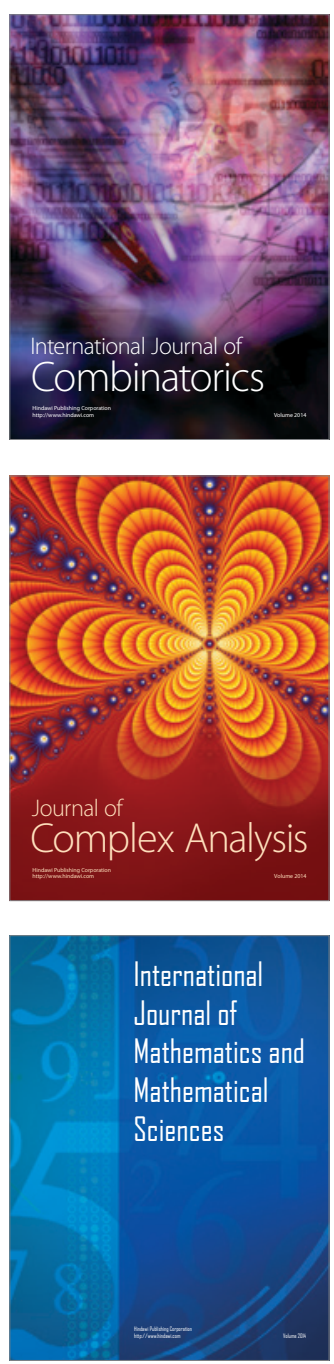
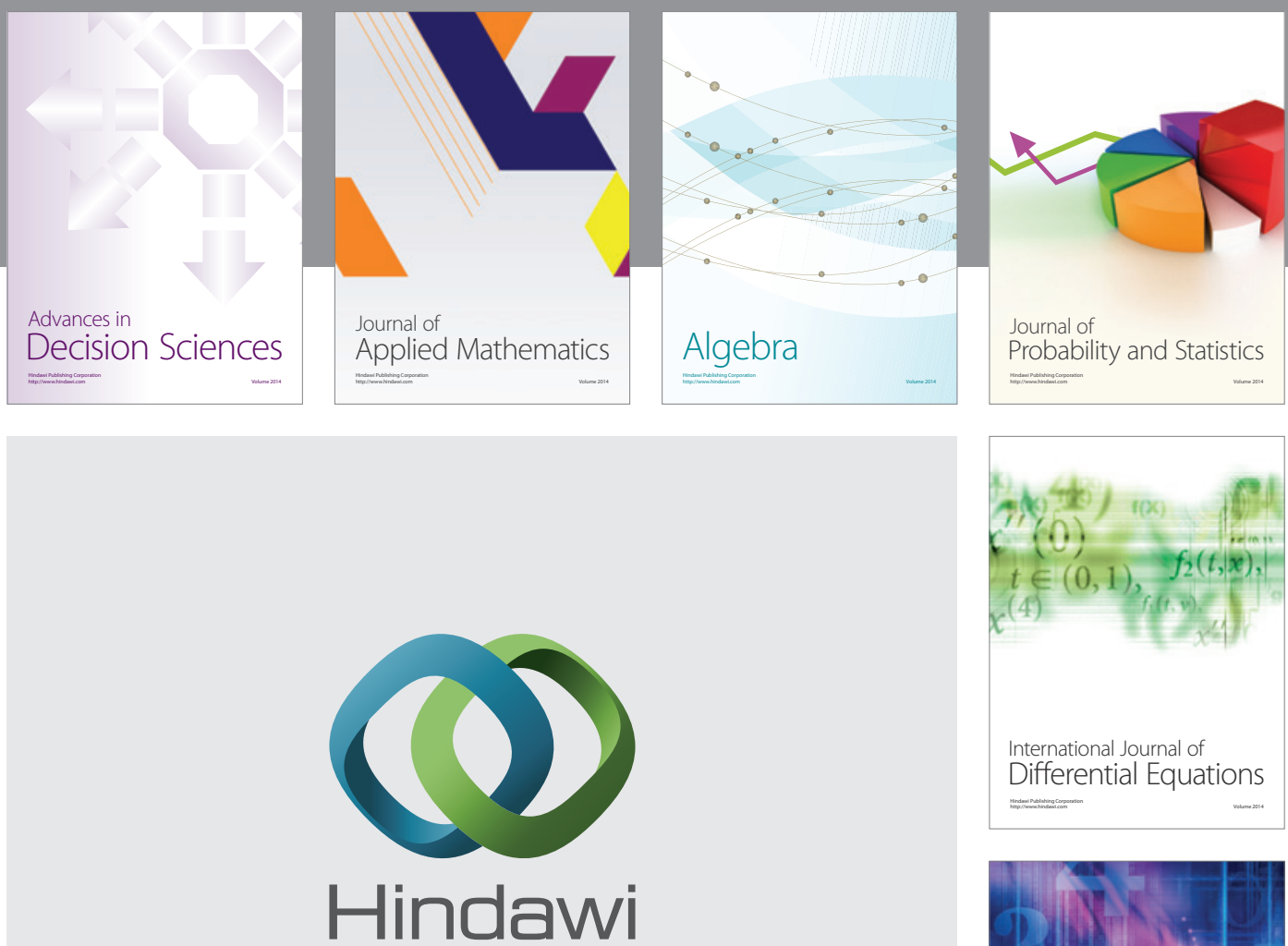

Submit your manuscripts at http://www.hindawi.com
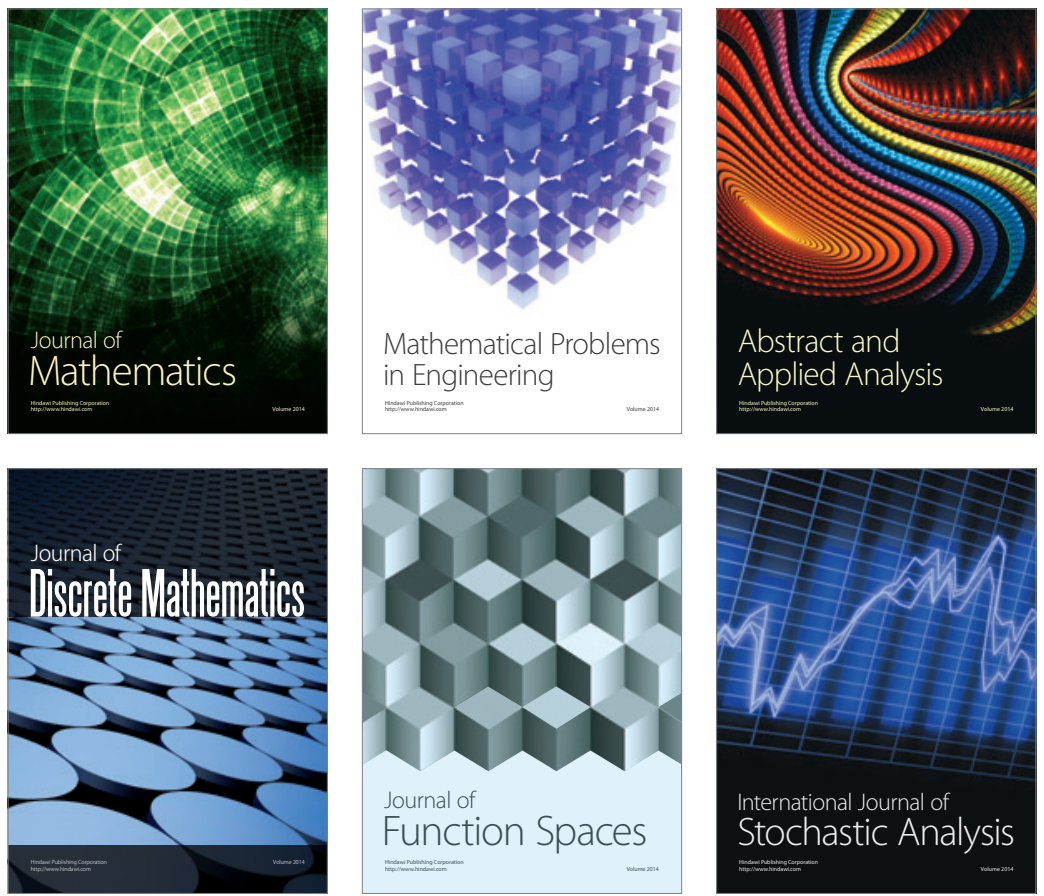

Journal of

Function Spaces

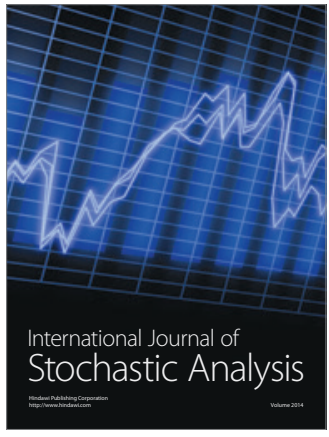

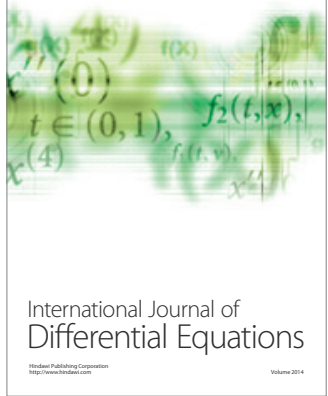
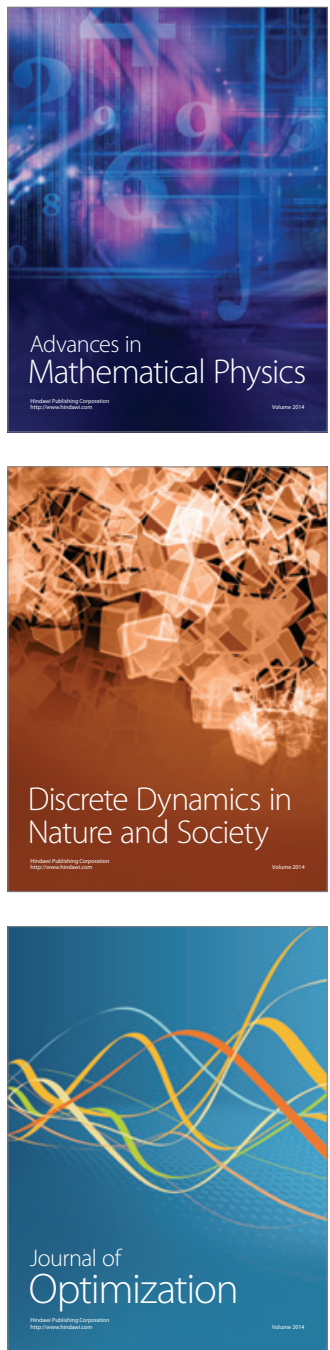